\title{
LOS RANKINGS DE LAS INSTITUCIONES DE EDUCACIÓN SUPERIOR: UNA REVISIÓN DEL PANORAMA INTERNACIONAL
}

\section{Introducción}

Los sistemas de educación superior de los países desarrollados están integrados por un gran número de centros e instituciones - universitarias y no universitarias, públicas y privadas- con características propias que las diferencian entre sí. El proceso de internacionalización y la "diversidad institucional" amplían la posibilidad de elegir el país al cual acudir, la universidad en la que estudiar, la titulación o el programa que cursar, la institución en que trabajar e investigar, entre otras libertades. Cada vez en un número mayor de países los interesados -estudiantes y sus familias, profesores, investigadores, empresas, profesionales, entre otros- acuden a toda una serie de rankings para que les orienten en la toma de decisiones.

Ahora bien, los rankings como tales llevan implícitos una determinada posición de cada institución que hace inevitable las comparaciones entre ellas. El problema surge cuando se identifica una mejor posición con una mejor calidad, dado el amplio espectro que abarca este concepto. Además, en el intento por medir y comparar la calidad debemos distinguir dos fenómenos: la evaluación llevada a cabo por las agencias de calidad de los distintos países y la elaboración de rankings universitarios, cuyo interés es, inicialmente, proveer información general a los estudiantes sobre las universidades a las que desean postular y a la sociedad en su conjunto.

Cualquier implicación que lleve a confundir estos dos fenómenos, bien distintos, es errónea. Es más, muchos autores y expertos en temas de calidad universitaria están de acuerdo en la necesidad de las primeras y no en el uso indiscriminado de rankings (Harvey, 2008). Por tanto, rankings y agencias existen por razones distintas y con objetivos diferenciados. Los primeros son elaborados 
para los medios masivos de divulgación y, por tanto, requieren ser fácilmente comprensibles y accesibles al público en general. No intentan ser exhaustivos en sus indagaciones sino más bien concretos, buscando un resultado final fácilmente interpretable. Por el contrario, las agencias de evaluación y acreditación intentan dar cuenta de una evaluación más exhaustiva de la calidad de las IES y están enfocadas como instrumento de gestión de los policy-makers de los distintos gobiernos (Fernández, Martínez y Velasco, 2006).

En la actualidad existe un gran número de rankings nacionales e internacionales de instituciones universitarias o de investigación que no se pueden obviar porque de forma directa o indirecta afectan a toda la comunidad universitaria. La elaboración de este tipo de jerarquizaciones se inició en Estados Unidos en 1983 y en el Reino Unido en 1990, casi de forma exclusiva para diferenciar a las mejores escuelas de negocios (Business School). Sin embargo, los académicos en particular y la sociedad en general les prestaron poca atención hasta comienzos del siglo XXI. En los últimos años han proliferado clasificaciones de instituciones de educación superior en América, Europa y en gran parte de los países desarrollados.

El impacto que, dentro y fuera de las IES, están ocasionando los rankings que se publican es cada vez mayor debido a su difusión por los medios de comunicación social. El análisis institucional de los rankings puede ayudarnos a contestar algunas de las preguntas clave: rankings, ¿para quién? ¿Por qué? y ¿qué? ¿Quién considera que son referencias importantes? ¿Los mejor posicionados? ¿Son los criterios suficientemente transparentes y objetivos? Y, ¿para qué pueden utilizarse en los distintos países? ¿Como indicador de excelencia y calidad universitaria? ¿Como una forma de canalizar la transparencia y la rendición de cuentas? ¿Como medida de financiación desde una perspectiva de política educativa?

El objetivo de este trabajo es doble: revisar las principales características y tendencias de los rankings internacionales y nacionales más importantes en el panorama actual de la educación superior y avanzar en una reflexión sobre las estrategias de futuro, cuya base es el análisis sobre debilidades, amenazas, fortalezas y oportunidades 
(DAFO) de los rankings de las IES. Por último, a modo de conclusión, se plantean unas reflexiones finales.

\section{Características y distintas clasificaciones de rankings de instituciones de educación superior}

Los rankings universitarios tienen por objeto realizar una jerarquización de las IES basándose en parámetros e indicadores que intentan medir la calidad de la educación universitaria, el grado de investigación y otros aspectos de la actividad académica, con el fin de informar y orientar a los estudiantes, a la opinión pública y a la sociedad en general.

En la actualidad existe una multitud de rankings de entidades de todo el mundo sobre los estudios de grado (undergraduate rankings), de posgrado (graduate programs and discipline rankings) y sobre otros aspectos (investigación, transferencia o su respuesta al "compromiso social"). Así, la elaboración de rankings universitarios y tablas de clasificación ${ }^{2}$ ya no es sólo exclusiva de países anglosajones.

Muchos de los rankings actuales están basados en indicadores de investigación y de docencia en páginas web institucionales. La información que suele utilizarse para su elaboración es de tipo cuantitativo y procede de: datos originarios de las estadísticas nacionales (ofrecidas por los propios gobiernos y sus administraciones o las agencias); datos internos de las IES, datos de publicaciones y citas. En ocasiones se complementa con información cualitativa procedente de las opiniones de expertos y encuestas a stakeholders (grupos de interés o partes interesadas).

En la mayoría de los casos los rankings no son oficiales sino financiados y publicados por organizaciones comerciales (en

2 En la terminología anglosajona se utilizan prácticamente como sinónimos los términos rankings y league tables. Para algunos autores (por ejemplo, Dill y Soo, 2005) los rankings de universidades se publican con un objetivo comercial. En nuestra opinión, se puede matizar que las tablas de clasificación (league tables) se basan en varios indicadores sin llegar a dar usualmente un ranking (nota final o número de orden), mientras que los rankings suelen ofrecer una posición, una medida ponderada mediante la elaboración de un indicador sintético. 
periódicos y semanarios ${ }^{3}$ ). En otros se trata de gabinetes de estudios o de consultoría para sociedades o empresas privadas, organizaciones no gubernamentales, así como también agencias gubernamentales -en una minoría- o incluso pueden estar dirigidos desde las universidades.

Cada ranking se elabora con su metodología particular, por lo que no son directamente comparables, aunque se tienda inevitablemente a su confrontación y, por tanto, generen cierta confusión. Los criterios utilizados pueden ser evaluados en función de su validez, completitud, relevancia, comprensión y funcionalidad (fiabilidad). Una elaboración compleja, en la que intervienen numerosos factores, pero en la cual es posible interpretar aquello que, en principio, puede parecer una simple clasificación numérica. A partir de las encuestas realizadas ${ }^{4}$ y las fuentes de información utilizadas se presenta un conjunto de indicadores de cuya categorización depende el resultado final.

Existen otros aspectos personales que pueden no estar bien reflejados en el ranking, como "el hecho de sentirse a gusto con el campus universitario, la localización, el costo o las ayudas financieras", y que incluso no se tienen en cuenta en muchos de los rankings que existen en la actualidad.

En todo caso, lo erróneo es considerar que los rankings son un mecanismo para medir la calidad de la educación superior, concepto que, lejos de ser claro, parece conducir a ciertas carencias en su medición, en particular por lo abstracto del término. La calidad no es un concepto uni sino multidimensional: calidad de la docencia, calidad de la investigación, calidad como una combinación de actividades, misión de las instituciones, entre otras dimensiones, todas ellas muy difíciles de agregar en un solo índice o ranking que

3 Aunque en muchas ocasiones se ha criticado a la prensa por publicar sus rankings sólo y exclusivamente con un objetivo comercial (básicamente para aumentar sus ventas), es cierto que la publicación de las clasificaciones en los periódicos ha forzado a muchas de las universidades (participantes y las que se han añadido posteriormente) a tomarse más en serio la recopilación de los datos y a generar una información mucho más transparente, cuidada y enriquecida.

4 Como señala el Informe C y D (2005) de la Fundación Conocimiento y Desarrollo, las encuestas a alumnos, graduados, profesores, "expertos" y "pares" introducen un grado de subjetividad adicional en los resultados. 
proporcione una "foto" completa del sector. Por otra parte, los rankings reflejan factores de "reputación" y no necesariamente la calidad y los resultados de las instituciones.

A pesar de todas las diferencias entre rankings (y su relación con la calidad), la mayoría de los indicadores (performance indicators) utilizados se suelen agrupar en tres bloques o grupos (Pérez-Esparrells y Salinas ${ }^{5}$, 1998): medidas de insumo (input measures) -por ejemplo, profesorado, alumnado, recursos económicos e instalaciones-, medidas de proceso (process measures) y medidas de resultados (output measures). Los indicadores dentro de cada bloque pueden ser muy diversos y su ponderación así como su influencia pueden variar ${ }^{6}$.

Los indicadores contemplados en cada uno de los bloques o grupos señalados son muy diversos, de tipo cuantitativo, y relacionados con la propia actividad universitaria. No obstante, pueden verse completados (en los rankings de carácter comercial) con otra serie de indicadores de carácter más cualitativo (reputación, prestigio, por ejemplo).

5 En Pérez Esparrells y Salinas (1998) se recoge una clasificación muy detallada de los indicadores de gestión en la evaluación de la calidad universitaria.

6 Como es bien sabido, para saber interpretar un ranking no basta con conocer en qué indicadores se ha basado sino que se necesita averiguar la ponderación de los mismos. 
Tabla 1

\begin{tabular}{|c|c|}
\hline \multicolumn{2}{|c|}{ Relación de indicadores empleados usualmente en la elaboración de rankings universitarios } \\
\hline Alumnos internacionales & Oferta optativa de la titulación \\
\hline Alumnos por profesor & Opinión de empleadores \\
\hline Antigüedad de la universidad en años & Opinión expertos \\
\hline Ayudas y becas & Perspectivas de los egresados \\
\hline Calidad de la investigación & Porcentaje de alumnos becados \\
\hline Calificación en las pruebas de acceso & Porcentaje de alumnos con Aporte Fiscal Indirecto \\
\hline Calificaciones de excelencia & Porcentaje de clases con 50 o más estudiantes. \\
\hline Citas por profesor & Porcentaje de clases con menos de 20 estudiantes. \\
\hline Colaboración internacional & Porcentaje de docentes con dedicación a jornada completa \\
\hline Completitud & Porcentaje de doctorados y de magíster \\
\hline Convenios internacionales & Porcentaje de donantes \\
\hline Créditos prácticos en empresas & Porcentaje de graduados (al cabo de 6 años) \\
\hline Dependencia privada o pública & Porcentaje de PDI a tiempo completo \\
\hline Docentes con posgrado & Porcentaje de permanencia tras el primer año \\
\hline Duración media de los estudios & Porcentaje de puntajes de excelencia en la Prueba de \\
\hline Educación para adultos & Selección Universitaria (PSU) \\
\hline Efectividad & Porcentaje en top 25 \\
\hline Empleabilidad & Porcentaje estudiantes de fuera de la provincia \\
\hline Encuestas externas & Porcentaje estudiantes internacionales \\
\hline Evaluación de la docencia & Porcentaje profesores doctores \\
\hline Evaluación de la investigación & Precio por crédito \\
\hline Excelencia de la docencia & Premios Nacionales \\
\hline Formación de magíster & Primer año de clases impartidas por profesores con \\
\hline Gasto corriente por alumno matriculado & experiencia \\
\hline Gasto en instalaciones & Producción citable o producción primaria. \\
\hline Gasto en libros y ordenadores & Producción de doctores \\
\hline Gasto medio por estudiante & Producción ponderada o potencial investigador. \\
\hline Grado académico de los docentes & Producción total \\
\hline Inclusividad & Profesores internacionales \\
\hline Índice de admisión & Proporción de estudiantes en relación al PDI \\
\hline Inversión en infraestructura & Proporción de estudiantes que realizan carreras largas \\
\hline Media de las calificaciones de High School & Proporción de estudiantes que terminan la carrera en los \\
\hline Metros cuadrados disponibles por alumno & años justos de carrera \\
\hline Nota de corte en el año en curso & Proporción de profesorado que son mujeres \\
\hline $\mathrm{N}^{0}$ de alumnos con aporte fiscal indirecto & Puestos en aulas/número de alumnos \\
\hline $\mathrm{N}^{0}$ de alumnos por computador & Puestos en laboratorios/número de alumnos \\
\hline $\mathrm{N}^{0}$ de alumnos por docente & Puntuación nivel A/AS \\
\hline $\mathrm{N}^{0}$ de centros con estudios de carreras largas & Ratio estudiante/personal (Student-staff ratio) \\
\hline $\mathrm{N}^{0}$ de citas por publicación & Ratio estudiantes/profesor \\
\hline $\mathrm{N}^{0}$ de créditos prácticos/teóricos & Salario medio del profesorado \\
\hline $\mathrm{N}^{0}$ de créditos totales de la titulación & Servicios a los estudiantes \\
\hline $\mathrm{N}^{0}$ de ejemplares en biblioteca & Tasa de abandono \\
\hline $\mathrm{N}^{0}$ de idiomas ofertado/obligatorio & Tasa de graduación \\
\hline $\mathrm{N}^{0}$ de plazas previstas & Tasa de participación de profesores en proyectos de \\
\hline $\mathrm{N}^{0}$ de proyectos de investigación & investigación \\
\hline $\mathrm{N}^{\circ}$ de publicaciones & Tasa de profesorado en relación con el número de \\
\hline $\mathrm{N}^{0}$ de puestos en bibliotecas/alumnos & estudiantes \\
\hline $\mathrm{N}^{0}$ de puestos en aulas de informática/alumnos & Tasa de profesorado en relación con el PAS \\
\hline $\mathrm{N}^{o}$ total de alumnos en la facultad y por curso & Tasa de tesis doctorales \\
\hline $\mathrm{N}^{o}$ total de publicaciones e impacto & \\
\hline
\end{tabular}

Fuente: elaboración propia. 


\section{Rankings de las IES en el ámbito nacional}

En la actualidad, en la mayoría de los países la preocupación por la calidad de los servicios que ofrecen las IES y la demanda de los usuarios por información "fiable" han provocado que surjan clasificaciones y jerarquizaciones que, con el tiempo, se van consolidando y permiten tomar decisiones fundadas.

Aunque es muy difícil establecer una clasificación general de los rankings universitarios se suele diferenciar entre uno de ámbito nacional y otro de ámbito internacional ${ }^{7}$. Se entiende por ámbito nacional cuando hace referencia sólo y exclusivamente a universidades/ escuelas de negocios del país de referencia. Asimismo, se califica como de ámbito internacional cuando abarca a universidades/escuelas de negocios de diferentes países y mundial cuando son analizados todos los países de forma global (overall rankings). No obstante, algunos rankings nacieron con la finalidad de referirse exclusivamente al ámbito nacional y con el paso de los años están tornando a un ámbito internacional.

En el medio nacional algunos de los rankings más importantes se refieren a los estudios de grado. Se corresponden con las siguientes áreas: América del Norte, América Latina, Europa (Reino Unido, Alemania, Francia, España e Italia) y Australia. Suelen clasificar las instituciones según cuatro categorías, para lo que se toma el Carnegie Classification System -National Universities, Liberal Arts Colleges, Universities-Master's, Baccalaureate Colleges- y se le aplica como criterios propios la misión de las instituciones y la naturaleza y tamaño de las mismas ${ }^{8}$.

7 Un análisis en detalle de los distintos rankings nacionales e internacionales puede encontrarse en Pérez Esparrells y López García (2009).

8 Se tiene en cuenta la diversidad de disciplinas así como el máximo grado de titulación de su oferta educativa. 
Tabla 2

Relación de rankings de ámbito nacional (estudios de grado)

América del Norte

- US News \& World Report (America's Best Colleges): 1.400 instituciones de educación superior, quince indicadores.

- The Maclean's Guide to Canadian (MacLean's Magazine): diecisiete indicadores.

América Latina

- Ranking revista "Qué Pasa": doce indicadores.

- Revista "El Sábado" (El Mercurio): diez indicadores.

Alemania

- CHE ranking, Centrum für Hochschulentwicklung (CHE, Centro para el Desarrollo de la Educación Superior): 280 instituciones de educación superior, cuarenta indicadores asociados a nueve dimensiones funcionales.

España

- Gaceta Universitaria (TuGueb): cuatro factores, once indicadores, 47 universidades públicas.

- El ranking de carreras del diario El Mundo (50 Carreras): veintiséis indicadores. 77 universidades públicas y privadas

Francia

- Le Nouvel Observateur (con Le Guide des École d'Ingénieurs y Le Palmarès des Universités): 16-25 indicadores.

- Semanario Le Point: Universités-Le Palmarès de la reussite, Écoles d’ingénieurs-Le Palmarès y Palmarès des grandes écoles): 16-25 indicadores para las universidades. Italia

- Il Sore 24 ORE, 3 bloques de indicadores, 60 universidades públicas y 16 privadas.

Reino Unido

- The Times Good University Guide (The Times), The Times top 100 Universities, Times Higher Education Supplement: 200 instituciones.

- The Sunday Times University Guide (The Sunday Times), The Sunday times university league.

- The Guardian University Guide: 183 instituciones.

Australia

- The Good University Guide: más de 100 instituciones.

- The International Standing of Australian Universities (Melbourne University): 30 universidades australianas.

Fuente: elaboración propia.

Existen otros rankings relativos a los estudios de posgrado y de escuelas de negocios (Business School), e incluso rankings vinculados con el compromiso social, que obviamente no son comparables directamente con los rankings de universidades. La especialización es la tónica dominante en el ámbito de las escuelas de negocios. La idea 
de que un centro es mejor que otro ha desaparecido para localizar la excelencia por ramas del saber e incluso por habilidades ${ }^{9}$.

Tabla 3

\begin{tabular}{|l|}
\hline Relación de otros rankings de ámbito nacional (estudios de posgrado) \\
\hline Estados Unidos: BusinessWeek, Newsweek, Forbes, Wall Street Journal, The Wall Street Journal. \\
\hline Francia: Libération. \\
\hline Australia: Hobsons. \\
\hline Reino Unido: The Economist, Financial Times, Guardian. \\
\hline Alemania: B-School Net. \\
\hline América Latina: América Economía. \\
\hline
\end{tabular}

Fuente: elaboración propia.

\section{Rankings de las IES de ámbito internacional}

Cada vez más, la tendencia en los rankings de carácter internacional es abarcar un mayor número de países, hasta llegar a elaborar rankings mundiales o globales (overall rankings). El problema es que resultan muy "generalistas" y sólo intentan medir la producción científica, la cual, por otra parte, es la única dimensión medible globalmente. Con unos criterios muy subjetivos, introducen una clasificación de las IES que corresponde a la idea "preconcebida" que se tiene de las mejores universidades en todo el mundo.

Entre los llamados rankings generalistas o mundiales destacan dos: el Shanghai Jiao Tong University ranking, conocido como ARWU (Academic Ranking of World Universities) y el Times Higher Education Supplement (THES-QS World Universities), utilizado inicialmente en EE.UU., China, Australia, Canadá y Reino Unido, pero que actualmente se han erigido como una referencia muy importante para todos los países. Asimismo, con la implantación del Espacio Europeo de Educación Superior están surgiendo cada vez más rankings que agrupan a las IES europeas.

9 Dado que se trata de una de las inversiones en capital humano más elevadas que se pueden realizar, los interesados, en gran parte procedentes de capas sociales más favorecidas económicamente, deben tener en cuenta a la hora de cursar un programa determinado más los subíndices y pesos utilizados que los genéricos, que pueden llevar en muchos casos a confusiones si no se saben interpretar o se desconoce la metodología. 
Cuadro 4

Relación de rankings de ámbito internacional (estudios de grado)

- Shanghai Jiao Tong University (China): 5000 universidades de todo el mundo.

- THES-QS World University Ranking, The Times Higher Education (Reino Unido): 5000 universidades de todo el mundo.

- Centro de Estudios de Ciencia y Tecnología (Centre for Science and Technology Studies, CWTS), Universidad de Leiden (Holanda): 1000 universidades, 4 rankings.

- Asiaweek's Best Universities, Asia.

- Rankings RI3 "Ranking Iberoamericano de Instituciones de Investigación", SCImago de la Universidad de Granada, España (Universia): nueve países iberoamericanos (España, México, Argentina, Brasil, Chile, Colombia, Portugal, Cuba y Venezuela): 766 instituciones.

- Ranking del Lisbon Council (Bruselas): quince países europeos (Reino Unido, Dinamarca, Finlandia, Suecia, Irlanda, Portugal, Italia, Francia, Polonia, Hungría, Holanda, Suiza, Alemania, Austria y España) y Estados Unidos y Australia.

- Centre for Higher Education Development (CHE): Ranking of Excellent European Graduate Programmes. Medallas.

Fuente: elaboración propia.

\section{Análisis DAFO para los rankings universitarios}

Uno de los objetivos principales del artículo es mostrar los distintos planteamientos sobre rankings universitarios realizados en diferentes países y conocer qué indicadores utilizan, con el fin de suscitar una reflexión sobre cómo analizar su aplicabilidad. Con esta finalidad, en esta sección se realiza un análisis DAFO (debilidades, amenazas, fortalezas y oportunidades) sobre los rankings universitarios, a partir de todos los indicadores señalados y revisados y de las ideas sugeridas por los distintos autores y expertos en la materia. La finalidad es plantear un diagnóstico interno en torno a las debilidades y fortalezas y un análisis externo en relación a las amenazas y oportunidades que influyen en la construcción y análisis de los rankings universitarios. 


\section{DEBILIDADES}

- Los rankings se construyen con indicadores de lo que puede ser medido, no con lo que es realmente relevante medir (Stella y Woodhouse, 2006), fundamentalmente debido a la escasez de datos homogéneos.

- No consideran la calidad de la docencia, ni la implantación de las nuevas metodologías docentes (Pulido, 2009), ni la política de contratación de académicos jóvenes.

- Están sesgados hacia la investigación y, en el caso de la investigación bibliométrica, hacia la que se publica en inglés (Altbach, 2006).

- Generalmente, no consideran el tamaño de las IES y queda patente que las universidades mejor posicionadas son, en general, las de mayor tamaño (López, Pérez-Esparrells y Montañez, 2008).

- Los pesos de los indicadores pueden ser arbitrarios y subjetivos, especialmente en los rankings de ámbito comercial, y varían continuamente en el tiempo (Stella y Woodhouse, 2006).

- A pesar del crecimiento espectacular de los rankings, algunas instituciones no están "deseosas" de participar en ellos, como es el caso de 19 universidades en China o de 11 instituciones en Canadá (Harvey, 2008).

\section{FORTALEZAS}

- La expansión global del acceso a la educación superior ha incrementado la demanda de información sobre la calidad de las IES y está contribuyendo al desarrollo de sistemas de rankings universitarios en muchos países del mundo (Fundación C y D, 2005).

- El impacto que, dentro y fuera de las IES, están ocasionando los rankings que se publican es cada vez mayor debido a su difusión por los medios de comunicación social.

- Una mayor información potencia las buenas prácticas (benchmarking) y facilita la decisión de movilidad de los estudiantes y de profesores e investigadores.

- Son más adecuados si permiten diferenciar entre diferentes tipos de IES, distintos programas y disciplinas (Meller y Rappaport, 2006), particularmente en el enfoque local/nacional (o de una determinada zona de influencia).

- Dentro de un país o área de influencia, los rankings se pueden elaborar para introducir financiamiento público basado en resultados de investigación o de docencia (Thakur, 2007).

- Los empleadores pueden utilizarlos a la hora de seleccionar la procedencia de sus futuros empleados (actualmente, está ocurriendo en EE.UU. y en el Reino Unido).

\section{AMENAZAS}

- Opinión de algunos escépticos que consideran que esta clase de rankings son un "sinsentido", tienen múltiples limitaciones y evalúan la calidad de manera "ingenua" (Harvey, 2008).

- Los rankings pueden implicar pérdida de libertad e independencia de las IES a la hora de establecer su "marca" o distintivo y controlar la medición de su éxito (Carey, 2006).

- Suponen una amenaza a la equidad porque pueden limitar el acceso a los estudiantes "poco aventajados" (disadvantage students). También se comprueba que son utilizados para seleccionar las IES sólo por parte de los estudiantes procedentes de familias de mayores ingresos o mejor educadas (Harvey, 2008). 
OPORTUNIDADES

- Elaborados con criterios transparentes, pueden servir para la rendición de cuentas a la sociedad (accountability).

- Para el reclutamiento de estudiantes extranjeros, pueden ser una buena carta de presentación de las IES mejor posicionadas en los rankings (Do Campo, 2008).

- Bien utilizados, pueden estimular la competencia entre las IES y se pueden plantear estrategias de futuro de cooperación entre universidades (Pérez-Esparrells y López García, 2009).

- Pueden incentivar la fusión de universidades para hacerse más grandes y tomar posiciones en los rankings, cuestión que de otra manera sería inviable (Pérez-Esparrells y López García, 2009).

- Se pueden utilizar como herramientas para evaluar una parte de la calidad (quizás la calidad de la investigación), completando el trabajo realizado por las agencias de evaluación de la calidad.

- Pueden generar un debate para contribuir a la definición de calidad de la educación superior en los ámbitos nacionales o de zonas de influencia (América, Europa, Australia, Asia, por ejemplo).

Fuente: elaboración propia.

\section{Conclusiones}

Como hemos visto, existen multitud de formas de clasificar los rankings universitarios: de ámbito nacional o internacional, generalistas o específicos, elaborados por instituciones públicas o privadas, o vinculados con el compromiso social. Por una parte, lo que ha quedado patente a lo largo de este artículo son dos hechos. Primero, la expansión global del acceso a la educación superior ha incrementado la demanda de información sobre la calidad de las IES y está contribuyendo al desarrollo de sistemas de rankings universitarios en muchos países del mundo. Segundo, el interés por la investigación de los sistemas de rankings universitarios y su depuración, ya que pueden suponer una contribución muy valiosa al desarrollo del mercado internacional de la educación superior.

Ahora bien, las fuentes de financiación, los patrones de gobernabilidad, el nivel de autonomía de la dirección, las diferencias entre las misiones institucionales, la disponibilidad de datos fiables y el potencial para manipular estadísticas son dimensiones importantes que contribuyen a la variación y apoyan algunas corrientes ideológicas que abogan por posicionarse en contra de la elaboración de rankings internacionales que midan a todas las instituciones con las mismas reglas. El Comité sobre Educación Superior e Investigación (Higher Education and Research Standing Committee) en Oslo estableció varias objeciones (Education International, 2006): los rankings son 
"irreconciliables" con el principio de equidad y las misiones de la educación, las cuales reflejan las diferentes aspiraciones de los estudiantes; llevan a concepciones "erróneas", etiquetando algunas instituciones como malas y creando élites; se niega que las IES puedan operar en entornos diferentes cultural, histórica y socialmente; animan a las instituciones de élite a elevar sus precios (tuition fees), lo cual es incompatible con la equidad, e intrínsecamente están ligados a la "comercialización" de la educación superior.

Asimismo, la Asociación de Estudiantes Europeos (European Students' Union, ESU, 2008) también señala que los rankings no son realmente sistemas para informar a los estudiantes (aunque supuestamente éste es su objetivo principal), sino que están diseñados para crear una jerarquía en términos de reputación y atraer así a los mejores talentos de todo el mundo. En este sentido, los rankings conducen a que las IES se preocupen demasiado por aquellos aspectos que son tenidos en cuenta en las metodologías de elaboración de los rankings, las cuales no incluyen en ningún caso los nuevos procesos de aprendizaje que, sin duda, están cambiando las políticas de las universidades de todos los países occidentales.

Sin embargo, a pesar de estas críticas y reticencias, la realidad es muy distinta, ya que los rankings internacionales están siendo cada vez más utilizados para comparar instituciones y están teniendo consecuencias en el incremento de la competencia entre las universidades, en el ámbito nacional y, en mayor medida, en el internacional entre las IES de más prestigio.

A pesar de su naturaleza tan controvertida e incluso discutible, hemos tenido ocasión de comprobar que se elaboran en más países y con una fuerza e influencia cada vez mayor en estudiantes, familias, empresarios, profesionales, organizaciones educativas, organismos independientes o sin ánimo de lucro, gobiernos e incluso las propias universidades.

También se ha puesto de manifiesto que la importancia dada a las clasificaciones varía sensiblemente de un país a otro, y la manera en que los gobiernos y los organismos académicos responsables de las instituciones de educación superior responden es muy diversa. 
En cuanto a la metodología de los rankings, éstos son aproximaciones a una realidad compleja y multidimensional. En general, los análisis multidimensionales pueden ser más convenientes que un solo indicador que refleje una posición global respecto de la calidad, especialmente cuando se trata de clasificar y comparar instituciones con diferentes actividades y diversas misiones. Por consiguiente, debe valorarse la información detallada que contienen por parte de todos los implicados: administraciones educativas, universidades, profesores, estudiantes, empresarios, profesionales, organismos independientes, entre otros, quienes a su vez tendrán distintos objetivos, por un lado, e intereses comunes y diferentes, por otro.

Los criterios sobre qué medir, los indicadores seleccionados y su peso relativo pueden cambiar radicalmente la valoración de las universidades con criterios de calidad. No sólo eso, los resultados de los rankings son muy sensibles a variaciones en la metodología y en los datos utilizados y, por tanto, en el medio nacional, se debe dar prioridad a la recopilación de fuentes de información adecuadas. Ahora bien, a nuestro modo de ver, con independencia de quiénes sean los "encargados de elaborar" los rankings nacionales, deberían considerarse tres aspectos clave: las ordenaciones para el conjunto de una universidad deberían desagregarse por centros, facultades, titulaciones, para evitar promedios que pueden añadir confusión si no se conocen los detalles (Pulido, 2009); se debería valorar no sólo la actividad investigadora -sesgo que se reproduce en la mayoría de los rankings internacionales-, sino también la actividad docente; por último, se debería tener en cuenta también el tamaño de los centros, para no caer en una de las "fallas" de algunos de los más prestigiosos rankings actuales, ya que se demuestra que el tamaño también importa en términos de mejor posicionamiento de la institución en el plano mundial.

En nuestra opinión, sería deseable incentivar iniciativas también en el medio internacional, pero circunscritas a un espacio geográfico (por ejemplo, el Espacio Europeo de Educación Superior) o a una lengua común (países de América Latina). De esta forma, se potenciaría la elaboración de un catálogo permanente de indicadores, 
no sólo en el ámbito institucional sino también en el de centros, facultades o titulaciones, efecto que tendría una triple utilidad, a nuestro modo de ver. En el plano internacional, puesto que permitiría relanzar en el campo de la docencia y la investigación a las universidades de habla hispana; en el nacional, puesto que podría permitir, en última instancia, una definición de políticas universitarias y estrategias de posicionamiento regionales; en el interno, porque permitiría a las propias universidades llevar a cabo una mejor gestión institucional y control, tanto mediante el análisis de los datos de sus propios centros, como para disponer de su evolución en el tiempo o permitir su comparación con otras universidades análogas, a modo de benchmarking en el mercado "hispanoamericano" de la educación superior.

Finalmente, las consecuencias de estos rankings y tablas de clasificación comienzan a ser muy amplias e influyen actualmente en las políticas de muchas IES, escuelas de negocios y centros de investigación de excelencia de todo el mundo. Sin embargo, cabe preguntarse si las IES pequeñas, de menor prestigio o localizadas en zonas o países menos desarrollados, deberían hacerse un hueco entre los grandes rankings que premian la investigación o si deberían centrarse en su docencia, las ayudas y becas al estudio y la investigación en el ámbito local, como apunta Harvey (2008).

\section{Referencias bibliográficas}

Altbach, P.G. (2006) The dilemmas of ranking. Bridges, 12.

Carey, K. (2006) Collage rankings reformed: the case for a new order in Higher Education. Education Sector Reports, 19.

Dill, D. \& Soo, M. (2005) Academic quality, league tables, and public policy: A Cross-National Analysis of University Ranking Systems. Higher Education, 49(4), pp. 495-534.

Docampo, D. (2008) Rankings internacionales y calidad de los sistemas universitarios. Revista de Educación, número extraordinario, pp. 149176.

Fernández, R.; Martínez, Y. y Velasco, N. (2006) Indicadores y estándares internacionales de calidad. Calidad en la Educación, 25, pp. 15-30. 
Fundación Conocimiento y Desarrollo (2005) La Contribución de las Universidades Españolas al Desarrollo, Informe C y D 2005.

Harvey, L. (2008) Ranking of Higher Education Institutions: A Critical Review! Quality in Higher Education, 14(3), pp. 187-207.

López, A.M. y Pérez-Esparrells, C. (2007) Los rankings universitarios: estado de la cuestión y posibles aplicaciones al caso español. Investigaciones de Economía de la Educación, 2, pp. 109-119.

López, A.M.; Pérez-Esparrells, C. y Montañez, M. (2008) Una aproximación a un indicador sintético para la evaluación del sistema universitario público español. Investigaciones de Economía de la Educación, 3, pp. 105-114.

Meller, P. y Rappoport, D. (2006) Nueva metodología para un ranking de universidades chilenas. Calidad en la educación, 25, pp. 55-78.

Pérez-Esparrells, C. y López, A.M. (2009) Estado de la cuestión de los rankings universitarios nacionales e internacionales. Documento UNIVNOVA, DU04, marzo.

Pérez-Esparrells, C. y Salinas, J. (1998) El uso de los indicadores de gestión en la evaluación de la calidad universitaria. Hacienda Pública Española, Monográfico Educación y Economía, pp. 157-167.

Pulido, A. (2009) El futuro de la Universidad. Un tema para debate dentro y fuera de las universidades, Madrid, Delta Publicaciones.

Stella, A. y Woodhouse, D. (2006) Ranking of Higher Education Institutions. Occasional Publications Series, 6, Melbourne, AUQA.

Thakur, M. (2007) The impact of ranking systems on Higher Education and its stakeholders. Journal of Institutional Research, 13(1), pp. 83-96.

Usher, A., Savino, M. (2007) A Global Survey of University Ranking and League Tables. Higher Education in Europe, 32, 1, pp. 5-15.

Recibido: 27 de mayo de 2009

Aceptado: 29 de mayo de 2009 